\title{
Integrating Systemic Risk and Risk Analysis Using Copulas
}

\author{
Stefan Hochrainer-Stigler ${ }^{1}$ - Georg Pflug ${ }^{1,2}$ • Ulf Dieckmann ${ }^{1}$ - Elena Rovenskaya ${ }^{1,4}$. \\ Stefan Thurner ${ }^{1,3,5,6} \cdot$ Sebastian Poledna $^{1,3}$ - Gergely Boza ${ }^{1,7}$ - Joanne Linnerooth-Bayer ${ }^{1}$. \\ Åke Brännström ${ }^{1,8}$
}

Published online: 14 December 2018

(C) The Author(s) 2018

\begin{abstract}
Systemic risk research is gaining traction across diverse disciplinary research communities, but has as yet not been strongly linked to traditional, well-established risk analysis research. This is due in part to the fact that systemic risk research focuses on the connection of elements within a system, while risk analysis research focuses more on individual risk to single elements. We therefore investigate how current systemic risk research can be related to traditional risk analysis approaches from a conceptual as well as an empirical point of view. Based on Sklar's Theorem, which provides a one-to-one relationship between multivariate distributions and copulas, we suggest a reframing of the concept of copulas based on a network perspective. This provides a promising way forward for integrating individual risk (in the form of probability distributions) and systemic risk (in the form of copulas
\end{abstract}

Stefan Hochrainer-Stigler

hochrain@iiasa.ac.at

1 International Institute for Applied Systems Analysis (IIASA), 2361 Laxenburg, Austria

2 Department of Statistics and Operations Research, University of Vienna, 1010 Vienna, Austria

3 Complexity Science Hub, 1080 Vienna, Austria

4 Faculty of Computational Mathematics and Cybernetics, Lomonosov Moscow State University, Moscow, Russia 119234

5 Medical University of Vienna, 1090 Vienna, Austria

6 Santa Fe Institute, Santa Fe, NM 87501, USA

7 Evolutionary Systems Research Group, MTA-ÖK-BLI Centre for Ecological Research, Hungarian Academy of Sciences, Tihany 8237, Hungary

8 Department of Mathematics and Mathematical Statistics, Umeå University, 90187 Umeå, Sweden describing the dependencies among such distributions) across research domains. Copulas can link continuous node states, characterizing individual risks, with a gradual dependency of the coupling strength between nodes on their states, characterizing systemic risk. When copulas are used for describing such refined coupling between nodes, they can provide a more accurate quantification of a system's network structure. This enables more realistic systemic risk assessments, and is especially useful when extreme events (that occur at low probabilities, but have high impacts) affect a system's nodes. In this way, copulas can be informative in measuring and quantifying changes in systemic risk and therefore be helpful in its management. We discuss the advantages and limitations of copulas for integrative risk analyses from the perspectives of modeling, measurement, and management.

Keywords Copulas · Individual risk · Risk analysis $\cdot$ Systemic risk

\section{Introduction}

Systemic risk is gaining increasing attention in theoretical and applied science disciplines due to the growing complexity of the world and the rise in data availability (Page 2015). The recent global risk report published by the World Economic Forum (WEF 2018) highlights the growing vulnerability to systemic risks and the limited understanding of its management, today and in the future. While research on systemic risk is not new-it has been discussed in ecology, for example, since the 1970s (Scheffer and Carpenter 2003)-recent events, in particular the global financial crisis of 2007/08, have increased interest on the part of researchers and practitioners to unprecedented 
levels (Boss et al. 2004; Thurner and Poledna 2013; Hochrainer-Stigler et al. 2018). A distinguishing feature of systemic risk is its emphasis on the connection between individual risks; it is therefore also called network risk (Helbing 2013). Contrary to systemic risk, individual risk focuses on single elements. Importantly, while the realization of individual risks may lead to a disaster in part of the system, the realization of systemic risk, by definition, leads to a breakdown, or at least a major dysfunction, of the whole system (Kovacevic et al. 2014).

Given the importance of assessing current and emerging systemic risks-be it in relation to ecological, financial, or socioeconomic systems (Centeno et al. 2015)-it is worthwhile investigating how systemic risk research can be related conceptually as well as empirically to well-established risk analysis approaches (Aven 2016). Systemic risk research focuses on the interdependencies of elements within a network (Helbing 2013). The failure of one or more of the interdependent elements can cause cascading effects throughout the network, eventually leading to system malfunction or collapse. In contrast, risk analysis focuses on probabilities of events, with probability distributions typically serving as general representations of risk (Pflug and Römisch 2007). Based on these observations, we address the question how the two disciplinary focal points, interdependencies and probabilities, can be merged. We suggest that the concept of copulas (Nelsen 2006), originally used for modeling multivariate distributions, can provide an answer to this question.

In general, a system or network contains elements that are interconnected. These elements can be "at risk," understood here as individual risk. A copula approach can model the interdependencies among the elements within the network, in combination with the risk of the individual elements described by distributions. Therefore, copulas can provide an integrative perspective. More formally, our starting point is Sklar's Theorem (1959), which provides a one-to-one relationship between multivariate distributions and copulas. Based on Sklar's Theorem, a copula enables the separation of a multivariate distribution into its marginal distributions (describing the individual risks in a standardized form, see Eq. 1) and the copula (describing the coupling of the individual risks in a standardized form, see Eq. 1). Taking a network perspective, we interpret the marginal distributions as describing the individual risks to continuous node states and the copula as describing the dependency between these node states within the network.

Apart from the benefit of enabling an integrated and standardized analysis of individual risk and systemic risk, we further argue that a copula approach can bring more realism to systemic risk models. As a case in point, gradual degradation processes and gradual dependencies are often neglected in systemic risk research, but are common in the real world. In ecology, for example, species extinction manifests as a gradual degradation process of the species' biomass (Borrvall et al. 2000). In finance, banks can experience gradual increases in stress eventually leading to default (May and Arinaminpathy 2010). In the natural hazard domain, catastrophic events are graduated in their severity (Grossi and Kunreuther 2005). These examples clearly underscore the widespread importance of gradual degradation processes. Even more importantly, degradation is often contingent on the severity of the disturbance of other elements within the network. For example, while non-extreme water discharge levels across European river basins have been shown to be independent of each other, it was recently confirmed that large-scale atmospheric processes can result in strongly correlated extreme discharges across river basins, leading to massive flooding (Jongman et al. 2014). In food webs, interactions between predators and prey follow a nonlinear process and are dependent on gradual changes of species' biomasses within the network (McCann 2000; Folke et al. 2004). Given these observations, we propose that a copula approach provides a promising way forward across research domains for a more refined description of coupling between nodes, and consequently, for a more realistic systemic risk analysis, because it can combine continuous node states (via the individual risk distribution of each element) with gradual degradation processes that are contingent on such node states (via a copula model). In the following, we provide a discussion of the advantages and limitations of copulas for integrating individual risk and systemic risk in terms of modeling, measurement, and management.

\section{Copulas to Model Systemic Risk}

Copulas originate from probability theory. From a statistical point of view, copulas are useful for modeling multivariate distributions of continuous random variables. The copula method goes back to Sklar's Theorem $(1959,1973)$, which states that the joint distribution function $H$ of any continuous random variables $X$ and $Y$ can be written as

$H(x, y)=C\left(F_{\mathrm{X}}(x), F_{\mathrm{Y}}(y)\right) \quad x, y \in \mathbb{R}$

with marginal probability distributions $F_{\mathrm{X}}(x)$ and $F_{\mathrm{Y}}(y)$ and (two-dimensional) copula $C:[0,1]^{2} \rightarrow[0,1]$. If $F_{\mathrm{X}}$ and $F_{\mathrm{Y}}$ are continuous, $C$ is uniquely defined. The functions $F_{\mathrm{X}}$ and $F_{\mathrm{Y}}$ are also known as cumulative distribution functions, and their inverse functions are known as quantile functions. While the copula contains the information about the structure of the dependency in a neatly standardized form across the unit square, the marginal distributions contain the information about the individual risks. There are many different copula types (Gaussian, Clayton, 
Fig. 1 Individual risks of nodes 1 and 2 (described by gamma distributions and their quantile functions) and their interdependency (described by the Joe copula). Dots in the scatterplot can be observed as joint observations of the states of nodes 1 and 2 . The density of dots across the unit square indicates the copula values, and the blue lines show the corresponding copula contours. The increasing tightness of the connection between the nodes (evidenced by the higher and more narrowly concentrated copula values in the upper right corner compared to the lower left corner) is highlighted by considering two ranges of disturbance levels (normal events in green and extreme events in red)
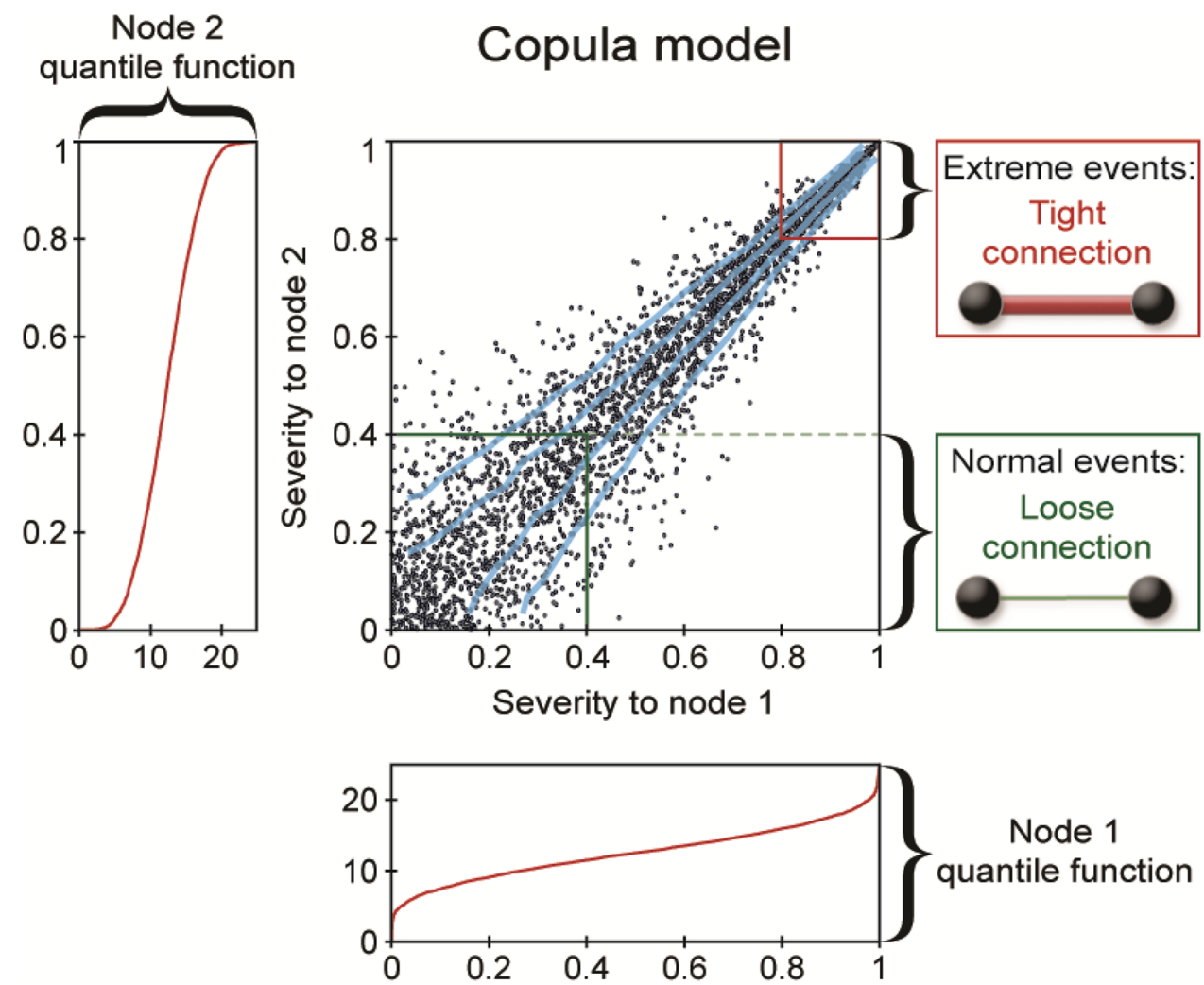

Gumbel, Frank, and Joe-to mention just a few), each describing different types of dependence structures, including independence (McNeil et al. 2015). In multivariate copula models (that is, with more than two random variables), there are several methods for structuring the dependency of random variables, including minimax approaches or vines (Bedford and Cooke 2002; Kurowicka and Cooke 2006; Kurowicka and Joe 2010; Timonina et al. 2015). It is beyond the scope of this article to discuss these different copula types in detail, and we refer the interested reader to Nelsen (2006) and McNeil et al. (2015).

For the purpose of illustration, we consider a network that consists of seven nodes, each being exposed to individual risk to its node state characterized by a gamma distribution (with mean 10 and variance 20). We assume for simplicity that this risk is a downside risk, that is, it represents loss or degradation (for example, biomass loss or monetary loss). We assume further that nodes are coupled by a Joe copula to describe increasing tail dependency (through Kendall's rank correlation coefficient $\rho$, see for example Hofert and Mächler 2011) in the system. Figure 1 shows how individual risks of two nodes are linked through the bivariate Joe copula ${ }^{1}$ for $\rho=0.8$ (for other values of $\rho$, see the row of panels at the top of Fig. 2)

\footnotetext{
${ }^{1}$ For the definition of the bivariate and the multivariate Joe copula, see Joe (2014).
}

For two nodes, the corresponding quantile functions are shown in Fig. 1. As in Eq. 1, the quantile function of the nodes are coupled through the copula. Due to the nature of the Joe copula, as seen in Fig. 1, lower quantiles show loose dependency, while higher quantiles show tight dependency. For normal events affecting nodes 1 and 2 (lower left corner of the scatterplot), having low severity and occurring with high probability, the copula indicates a loose connection between them. For extreme events (upper right corner of the scatterplot), having high severity and occurring with low probability, the copula indicates a tight connection between them. Depending on the research domain, the tighter coupling can be interpreted, for example, as a higher risk of spreading when diseases are becoming particularly prevalent or as a higher dependency when natural hazard-induced disasters are particularly severe or the default of banks results from particularly large monetary losses. A copula can model such contingent nonlinear dependencies between individual risks.

As a statistical concept, a copula is instrumental if the underlying drivers and interactions among elements within a complex system are not well known, but increasing tail dependency is often expected for theoretical reasons or follows from empirical observations. The phenomenon of increasing tail dependency as shown in Fig. 1 is especially noticeable in physical processes such as water discharge levels across basins. In the work of Jongman et al. (2014), 


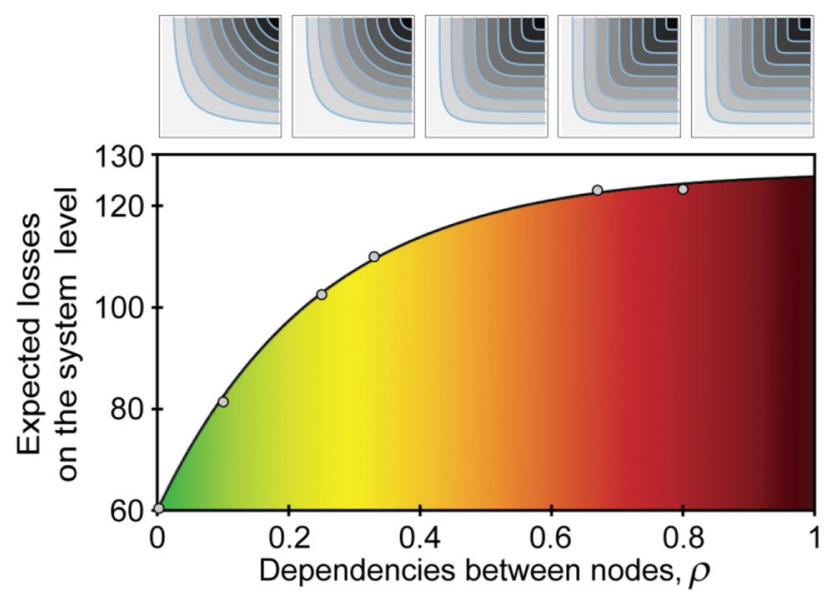

Fig. 2 Influence of a single-node failure on system-level losses for different copula-mediated dependencies between nodes. The row of panels at the top indicates the pairwise node coupling given by the Joe copula. The circles in the bottom panel indicate numerical results for different values of $\rho$, with the color shaded area summarizing how the expected losses are steeply rising with $\rho$

large-scale atmospheric processes can result in strongly correlated extreme discharges across river basins. While it is not possible to physically model these processes (due to data limitations and limited knowledge of key properties of river structures, such as sediments that affect water runoff), a copula approach circumvents this limitation by using empirical observations (Timonina et al. 2015). This opens up new possibilities to model risk management strategies. For example, based on the findings in Jongman et al. (2014) and Timonina et al. (2015), new stress tests for flood financing instruments could be performed. This revealed a high susceptibility of one core financing instrument, the European Union Solidarity Fund, which faces a significant risk of depletion following extreme flood events (Hochrainer-Stigler et al. 2017).

The copula approach, however, may be of limited value for exploring causal links and corresponding emergent behavior that influence risk in complex systems. For example, the modeling of self-organized systems with tipping points - developed by Bak and Paczuski (1995) for large dynamic systems that can self-organize into highly interactive critical states in which even minor perturbations can lead to systemic events-cannot be accomplished through copula approaches alone and might instead call for approaches using agent-based modeling (ABM) (Allen and Gale 2000; Jain and Krishna 2001; Hanel et al. 2007; Battiston, Gatti et al. 2012). Copula approaches are also of limited value when nonlinearities within complex networks need to be at the focus of analyses, and again, ABM approaches are superior for investigating such behavior (Poledna and Thurner 2016; for an overall discussion, see Page 2015). Links between copula approaches and ABM approaches are feasible and potentially beneficial for realworld analyses that combine individual risk and systemic risk (see the discussion in Poledna and Thurner 2016).

\section{Copulas to Measure Systemic Risk}

Risk at the system level can be measured by combining individual risk estimates with a copula model for a given network. Importantly, the influence of the interdependencies between the individual risks on systemic risk can be assessed (Borgomeo et al. 2015; Jeong et al. 2016).

To illustrate this, we use the already introduced sevennode network model. Figure 2 illustrates how the failure of a single node in this network can trigger, owing to the existence of systemic risk, larger system-level losses as failures between coupled nodes spread depending on the dependency assumed for the coupling (as shown in the row of panels at the top of Fig. 2). Single nodes are here assumed to fail if node losses exceed a node's value at risk (VaR) at, for example, the 95\% level (which means that failures individually occur with $5 \%$ probability). With this assumption, we calculate the expected total system-level losses as

$\mathrm{E}\left(\sum_{i=2}^{7} L_{i} \mid L_{1} \geq \operatorname{VaR}_{0.95}\right)$,

where $\mathrm{E}$ indicates the expectation value. Figure 2 demonstrates how accounting for the copula-mediated dependency of nodes within a network can be critical for more accurately modeling total losses from a systems perspective. For the considered network, system-level losses would be underestimated with errors of more than $100 \%$ if the coupling between nodes were assumed to be constant instead of copula-mediated, that is, severity-dependent. Even for only moderate tail dependencies, as described by the dependency parameter $\rho$, total losses are rising steeply. In other words, with increasing tail dependency the risk of system wide large losses is much higher given that a large risk realizes in one specific node.

It is important to recognize that two networked systems may have a close resemblance in terms of their nodes and the individual probability distributions that characterize each node; yet, depending on the node interdependencies, the systems can be essentially different. This makes the detection of systemic risk especially difficult. Increases in individual risk may be less significant for the risk of the whole system than increases in their interdependency. Moreover, the dependency that triggers systemic risk may occur only in extreme cases, which means that it cannot be observed under normal circumstances (for illustration, see Fig. 1). Copulas can therefore be useful for measuring 
systemic risk. This is underscored by Pflug and Pichler (2018), who have used a copula approach to introduce a new measure of systemic risk defined by the difference in risk (for example, using the value at risk as a risk measure) on the system level between the independent and the interdependent cases.

While it was assumed for illustration in Fig. 2 that each node has the same individual probability distribution, copula approaches readily apply to systems in which these distributions differ among nodes. In this context, it is worth highlighting that the description of the dependency between nodes provided by a copula is independent of the absolute scales of the disturbances impacting the separate nodes, since copulas express the dependency based on quantiles. Hence, in some systems even disturbances of one node that are small compared to the system-level average might result in a tight coupling with another node in the network, which subsequently can result in large disturbances at the system level.

It is thus clear that copulas can be used both directly, serving as a measure of systemic risk (for example, through the dependency parameter and copula type), and indirectly, providing the basis for estimating other measures of systemic risk (for example, through assessing total losses under dependency). At the same time, copulas as measures of systemic risk have some shortcomings. For example, one of the most prominent systemic risk measures in finance today is the so-called DebtRank (Battiston, Puliga et al. 2012). DebtRank estimates the impact of one node on the others and is inspired by the notion of centrality in a network. The centrality of a node is defined by its distress on other nodes in the network, and DebtRank can be considered an early-warning indicator for being "too central to fail" (in addition to being "too big to fail"; see the discussion by Poledna and Thurner 2016). While some copula approaches, such as vine copula models and hierarchical copula models (McNeil et al. 2015), can be used to obtain a proxy of node centrality, they have limitations compared to measures such as DebtRank. In particular, copulas describing a node's direct impact on another node do not as such capture how additional indirect impacts on that same node may arise from effects rippling through the network. Yet a copula approach has an advantage over DebtRank in that it integrates measures of individual risk and systemic risk.

\section{Copulas to Inform the Management of Systemic Risk}

Finally, a copula approach can help with considering options for mitigating systemic risk. In the context of climate change, for example, a copula approach has proven potentially useful in modeling future climate-related disaster events by assuming different strengths of their interrelationships (for example, between drought duration and intensity) and in assessing the consequences of mitigation measures currently in place (Borgomeo et al. 2015; for other applications in this context, see Jeong et al. 2016). The most common risk mitigation option for financial risks (not accounting for systemic risk) is risk transfer through insurance (Haefeli and Liedtke 2012), and more generally, risk diversification (Kessler 2014), which is therefore our focal point for the brief discussion below.

In the context of systemic risk, it is often presumed that diversification is needed for system stability (Haldane and May 2011), a presumption that was analyzed in detail by Battiston, Gatti et al. (2012), as well as others (Gai and Kapadia 2010; Allen et al. 2012). However, when considering copula-mediated couplings, the picture is not so clear. Already in the 1970s, May (1972) and others (Gardner and Ashby 1970; Pimm and Lawton 1978) found contradictory results regarding diversification; in particular, diversity can also destabilize community dynamics (Tilman and Downing 1996; McCann 2000) and can lead to a higher probability of contagion in the system (Battiston, Gatti et al. 2012). Again using the previously mentioned seven-node network setup, Fig. 3 illustrates how diversification can aid

\begin{tabular}{|c|c|c|c|c|}
\hline Network & $\begin{array}{llll} & & 0 & \\
\bullet & & 0 \\
\bullet & & & 0 \\
& 0 & 0 & 0\end{array}$ & & 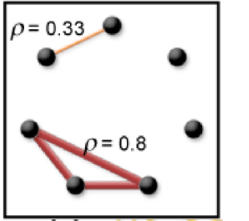 & $\begin{array}{c}\rho=0.33 \quad \rho=0.67 \\
\end{array}$ \\
\hline $\begin{array}{l}\text { Dependency } \\
\text { parameter }\end{array}$ & $\rho=0$ & $\rho=0.8$ & $\rho=0,0.33,0.8$ & $\begin{array}{c}\rho=0,0.33,0.67, \\
0.8\end{array}$ \\
\hline $\begin{array}{l}\text { System-level } \\
\text { losses }\end{array}$ & $V a R_{0.95}=60$ & $\operatorname{VaR}_{0.95}=129$ & $V_{a R_{0.95}}=101$ & $V a R_{0.95}=103$ \\
\hline
\end{tabular}

Fig. 3 Value at risk of system-level losses for different heterogeneous networks 
the management of systemic risk by modularizing the strengths of tail dependency.

Generally speaking, risk diversification via modularization can result in nonobvious changes in system-level risk (Helbing 2013). Fortunately, with a copula approach these changes are fully quantifiable, as shown in Fig. 3. We consider again the $95 \%$ value at risk (VaR) for systemlevel losses as a measure of systemic risk. The fully independent network $(\rho=0)$ results in $\operatorname{VaR}_{0.95}=60$, whereas with strong dependency $(\rho=0.8)$ this value increases to $V_{a} R_{0.95}=129$. For illustrative purposes, we compare the homogeneously connected network to modularized scenarios with heterogeneous changes in the copula dependency parameter. While various such scenarios could be looked at, the main importance is that all of them can be quantified and can be made comparable in a precise way. As Fig. 3 illustrates, systemic risk can be substantially decreased through modularizing dependency. But more importantly, one can precisely quantify the increase in system-level risk. For example, the two networks on the right in Fig. 3 show a $2 \%$ increase of system-level risk due to the increase of dependency between two previously independent nodes. Hence, the impacts of different diversification strategies (for example, in the form of modularizing dependency) can be precisely quantified. This could be especially useful for practical applications, for example, in cases in which some connectedness is considered desirable, but not at the cost of allowing systemic risk to rise above a critical level (Pflug and Pichler 2018).

\section{Conclusion}

It has recently been argued, for example, by Helbing (2013) that systemic risk can be better understood by moving from a component-oriented approach to an interaction- and network-oriented approach. Similarly, we have called for treating individual risks and systemic risks simultaneously, and have outlined how to utilize copulas for this purpose. In this way, traditional risk analyses and innovative systemic risk analyses can be further integrated. Additionally, copulas have the advantage of linking continuous node states through gradual dependency, so that models of systemic risk can become more realistic, more accurate, and more easily connected with traditional risk analysis approaches.

Despite these benefits, copula approaches are only one of many frameworks for systemic risk analysis, and especially process-based approaches, such as agent-based models (ABMs), have some advantages over copula approaches: in contrast to copula models, ABMs use causal models to derive emergent behavior at the system level, and importantly, they can model emergent nonlinear relationships. Nevertheless, we argue that copula models provide a good entry point, conceptually as well as empirically, for dealing with systemic risk from many disciplinary perspectives. Different scales of integration (from local to regional and global) can be examined with different forms of copulas. Hybrid frameworks that use copula approaches and ABM approaches together are possible, for example, by using copula-based results as an input to process-based approaches or by estimating copulas as informative outputs from process-based models even when not using them as model inputs. The most appropriate approach will always depend on the research question, system scale, data availability, and nature of the problem at hand.

As experienced in the past, the realization of systemic risk can have dramatic and possibly long-lasting impacts (Little 2002; WEF 2018). Thus, it is important to develop improved approaches for systemic risk analysis (Poledna and Thurner 2016). The copula approach as discussed here can advance current understanding and analyses of systemic risk by providing a holistic picture of risk, from the individual scale to the system scale.

Open Access This article is distributed under the terms of the Creative Commons Attribution 4.0 International License (http://crea tivecommons.org/licenses/by/4.0/), which permits unrestricted use, distribution, and reproduction in any medium, provided you give appropriate credit to the original author(s) and the source, provide a link to the Creative Commons license, and indicate if changes were made.

\section{References}

Allen, F., and D. Gale. 2000. Financial contagion. Journal of Political Economy 108(1): 1-33.

Allen, F., A. Babus, and E. Carletti. 2012. Asset commonality, debt maturity and systemic risk. Journal of Financial Economics 104(3): 519-534.

Aven, T. 2016. What defines us as professionals in the field of risk analysis? Risk Analysis 37(5): 854-860.

Bak, P., and M. Paczuski. 1995. Complexity, contingency, and criticality. Proceedings of the National Academy of Sciences of the USA 92(15): 6689-6696.

Battiston, S., D.D. Gatti, M. Gallegati, B. Greenwald, and J.E. Stiglitz. 2012. Liaisons dangereuses: Increasing connectivity, risk sharing, and systemic risk. Journal of Economic Dynamics and Control 36(8): 1121-1141.

Battiston, S., M. Puliga, R. Kaushik, P. Tasca, and G. Caldarelli. 2012. Debtrank: Too central to fail? Financial networks, the FED and systemic risk. Scientific Reports 2(8): Article 541.

Bedford, T., and R. Cooke. 2002. Vines: A new graphical model for dependent random variables. Annals of Statistics 30(4): 1031-1068.

Borgomeo, E., G. Pflug, J.W. Hall, and S. Hochrainer-Stigler. 2015. Assessing water resource system vulnerability to unprecedented hydrological drought using copulas to characterize drought 
duration and deficit. Water Resources Research 51(11): 8927-8948.

Borrvall, C., B. Ebenman, and T. Jonsson. 2000. Biodiversity lessens the risk of cascading extinction in model food webs. Ecology Letters 3(2): 131-136.

Boss, M., M. Summer, and S. Thurner. 2004. Contagion flow through banking networks. In Computational Science-ICCS 2004, Proceedings, Part III, ed. M. Bubak, G.D. van Albada, P.M.A. Sloot, and J. Dongarra, 1070-1077. Berlin: Springer.

Centeno, M.A., M. Nag, T.S. Patterson, A. Shaver, and A.J. Windawi. 2015. The emergence of global systemic risk. Annual Review of Sociology 41(1): 65-85.

Folke, C., S. Carpenter, B. Walker, M. Scheffer, T. Elmqvist, L. Gunderson, and C.S. Holling. 2004. Regime shifts, resilience, and biodiversity in ecosystem management. Annual Review of Ecology, Evolution, and Systematics 35(1): 557-581.

Gai, P., and S. Kapadia. 2010. Contagion in financial networks. Proceedings of the Royal Society A 466: 2401-2423.

Gardner, M.R., and W.R. Ashby. 1970. Connectance of large dynamic (cybernetic) systems: Critical values for stability. Nature 228(5273): 784.

Grossi, P., and H. Kunreuther (eds.). 2005. Catastrophe modeling: A new approach to managing risk. Boston, MA: Springer.

Haefeli, D., and P.M. Liedtke (eds.). 2012. Insurance and resolution in light of the systemic risk debate. Geneva, Switzerland: The Geneva Association.

Haldane, A.G., and R.M. May. 2011. Systemic risk in banking ecosystems. Nature 469(7330): 351-355.

Hanel, R., S.A. Kauffman, and S. Thurner. 2007. Towards a physics of evolution: Critical diversity dynamics at the edges of collapse and bursts of diversification. Physical Review E 76: Article 030101 .

Helbing, D. 2013. Globally networked risks and how to respond. Nature 497(7447): 51-59.

Hochrainer-Stigler, S., G. Boza, C. Colon, S. Poledna, E. Rovenskaya, and U. Dieckmann. 2018. Resilience of systems to individual risk and systemic risk. In Resource guide on resilience, Volume 2, ed. M.-V. Florin, and I. Linkov. Lausanne, Switzerland: EPFL International Risk Governance Center (IRGC).

Hochrainer-Stigler, S., J. Linnerooth-Bayer, and A. Lorant. 2017. The European Union Solidarity Fund: An assessment of its recent reforms. Mitigation and Adaptation Strategies for Global Change 22(4): 547-563.

Hofert, M., and M. Mächler. 2011. Nested Archimedean copulas meet R: The nacopula package. Journal of Statistical Software 39(9): $1-20$.

Jain, S., and S. Krishna. 2001. A model for the emergence of cooperation, interdependence, and structure in evolving networks. Proceedings of the National Academy of Sciences of the USA 98(2): 543-547.

Joe, H. 2014. Dependence modeling with copulas. Chapman and Hall/ CRC.

Jeong, B., W. Lee, D.S. Kim, and H. Shin. 2016. Copula-based approach to synthetic population generation. PLOS ONE 11(8): Article e0159496.

Jongman, B., S. Hochrainer-Stigler, L. Feyen, J.C.J.H. Aerts, R. Mechler, W.J.W. Botzen, and L.M. Bouwer, et al. 2014.
Increasing stress on disaster-risk finance due to large floods. Nature Climate Change 4(4): 264-268.

Kessler, D. 2014. Why (re)insurance is not systemic. Journal of Risk and Insurance 81(3): 477-488.

Kovacevic, R.M., G.C. Pflug, and A. Pichler. 2014. Measuring and managing risk. In Investment risk management, ed. H.K. Baker, and G. Filbeck, 17-41. Oxford: Oxford University Press.

Kurowicka, D., and R. Cooke. 2006. Uncertainty analysis with high dimensional dependence modelling. West Sussex, UK: Wiley.

Kurowicka, D., and H. Joe. 2010. Dependence modeling: Vine copula handbook. Singapore: World Scientific Publishing Co.

Little, R.G. 2002. Controlling cascading failure: Understanding the vulnerabilities of interconnected infrastructures. Journal of Urban Technology 9(1): 109-123.

May, R.M. 1972. Will a large complex system be stable? Nature 238(5364): 413-414.

May, R.M., and N. Arinaminpathy. 2010. Systemic risk: The dynamics of model banking systems. Journal of the Royal Society Interface 7(46): 823-838.

McCann, K.S. 2000. The diversity-stability debate. Nature 405(6783): 228-233.

McNeil, A.J., R. Frey, and P. Embrechts (eds.). 2015. Quantitative risk management: Concepts, techniques and tools. Princeton, NJ: Princeton University Press.

Nelsen, R.B. 2006. An introduction to copulas. New York: Springer.

Page, S.E. 2015. What sociologists should know about complexity. Annual Review of Sociology 41(1): 21-41.

Pimm, S.L., and J.H. Lawton. 1978. On feeding on more than one trophic level. Nature 275(5680): 542-544.

Pflug, G., and A. Pichler. 2018. Systemic risk and copula models. Central European Journal of Operations Research 26(2): 465-483.

Pflug, G.C., and W. Römisch. 2007. Modeling, measuring and managing risk. Singapore: World Scientific Publishing Co.

Poledna, S., and S. Thurner. 2016. Elimination of systemic risk in financial networks by means of a systemic risk transaction tax. Quantitative Finance 16(10): 1599-1613.

Scheffer, M., and S.R. Carpenter. 2003. Catastrophic regime shifts in ecosystems: Linking theory to observation. Trends in Ecology \& Evolution 18(12): 648-656.

Sklar, A. 1959. Distribution functions with $n$ dimensions and their margins (Fonctions de répartition à $n$ dimensions et leurs marges). Publications de l'Institut Statistique de l'Université de Paris 8: 229-231 (in French).

Sklar, A. 1973. Random variables, bivariate distribution functions and copulas. Kybernetika 9: 449-460.

Thurner, S., and S. Poledna. 2013. DebtRank-transparency: Controlling systemic risk in financial networks. Scientific Reports 3(5): Article 1888.

Tilman, D., and J.A. Downing. 1996. Biodiversity and stability in grasslands. In Ecosystem management, ed. F.B. Samson and F.L. Knopf, 3-7. New York: Springer.

Timonina, A., S. Hochrainer-Stigler, G. Pflug, B. Jongman, and R. Rojas. 2015. Structured coupling of probability loss distributions: Assessing joint flood risk in multiple river basins. Risk Analysis 35(11): 2102-2119.

WEF (World Economic Forum). 2018. The global risk report. Geneva, Switzerland: WEF. 